BASIC SCIENCE ARTICLE

\title{
Sarcomere gene variants act as a genetic trigger underlying the development of left ventricular noncompaction
}

Asami Takasaki ${ }^{1}$, Keiichi Hirono ${ }^{1}$, Yukiko Hata ${ }^{2}$, Ce Wang ${ }^{1,3}$, Masafumi Takeda ${ }^{4}$, Jun K Yamashita ${ }^{4}$, Bo Chang ${ }^{1}$, Hideyuki Nakaoka ${ }^{1}$, Mako Okabe ${ }^{1}$, Nariaki Miyao ${ }^{1}$, Kazuyoshi Saito ${ }^{1}$, Keijiro Ibuki ${ }^{1}$, Sayaka Ozawa ${ }^{1}$, Michikazu Sekine ${ }^{5}$, Naoki Yoshimura ${ }^{6}$, Naoki Nishida $^{2}$, Neil E. Bowles ${ }^{7}$ and Fukiko Ichida ${ }^{1}$

BACKGROUND: Left ventricular noncompaction (LVNC) is a primary cardiomyopathy with heterogeneous genetic origins. The aim of this study was to elucidate the role of sarcomere gene variants in the pathogenesis and prognosis of LVNC.

METHODS AND RESULTS: We screened 82 Japanese patients (0-35 years old), with a diagnosis of LVNC, for mutations in seven genes encoding sarcomere proteins, by direct DNA sequencing. We identified variants in a significant proportion of cases (27\%), which were associated with poor prognosis $(p=0.012)$, particularly variants in TPM1, TNNC1, and ACTC1 $(p=0.012)$. To elucidate the pathological role, we developed and studied human-induced pluripotent stem cells (hiPSCs) from a patient carrying a TPM1 p. Arg178His mutation, who underwent heart transplantation. These cells displayed pathological changes, with mislocalization of tropomyosin 1, causing disruption of the sarcomere structure in cardiomyocytes, and impaired calcium handling. Microarray analysis indicated that the TPM1 mutation resulted in the down-regulation of the expression of numerous genes involved in heart development, and positive regulation of cellular process, especially the calcium signaling pathway.

CONCLUSIONS: Sarcomere genes are implicated as genetic triggers in the development of LVNC, regulating the expression of numerous genes involved in heart development, or modifying the severity of disease.

Pediatric Research (2018) 84:733-742; https://doi.org/10.1038/s41390-018-0162-1

\section{INTRODUCTION}

Left ventricular noncompaction (LVNC) is thought to be due to an arrest of normal endomyocardial morphogenesis. ${ }^{1}$ The American Heart Association classifies LVNC as a primary cardiomyopathy with a genetic origin. ${ }^{2}$ The genetic basis of LVNC remains largely unknown, although a broad genetic spectrum for LVNC has gradually emerged in recent years: genes encoding taffazin (TAZ), cypher/ZASP or LIM domain-binding protein (LDB3), lamin $A / C$ $(L M N A)$, a-dystrobrevin (DTNA), and sodium channel type $\mathrm{Va}$ $(S C N 5 A){ }^{3}$ In addition, sarcomere gene variants, such as in myosin heavy chain 7 (MYH7), myosin-binding protein $C$ (MYBPC3), cardiac troponin $\mathrm{T}$ type 2 (TNNT2), a-cardiac actin 1 (ACTC1), and atropomyosin (TPM1), have also been identified in patients with LVNC. $^{4,5}$ Recently, we reported that next-generation sequencing reveals a high incidence of sarcomere gene variants, as well as variants in $T A Z$, in a young cohort of LVNC patients, with diagnosis ranging from during the fetal period to 12 years of age. ${ }^{6}$ However, understanding the molecular mechanism responsible for the development of LVNC due to sarcomeric variants is lacking.

An increasing number of reports of prenatal diagnosis of LVNC using fetal echocardiography support the notion that the arrest of myocardial morphogenesis and abnormal myocardial development underlies the pathogenesis of LVNC. We have previously reported the prenatal diagnosis of LVNC during the second trimester, associated with a novel mutation in $\mathrm{MYH7.}{ }^{7}$ Therefore, we hypothesized that sarcomere gene variants may alter normal maturation and function of the sarcomere, which is crucial during cardiac development and LV compaction during fetal life, ${ }^{8}$ and may have a pivotal role in inducing depressed LV function after birth leading to a poor prognosis.

In this study, we performed a systematic screening for mutations, focusing on seven sarcomere genes, in a large cohort of young patients with LVNC, and investigated the impact of sarcomere gene mutations on clinical phenotypes. In addition, we studied patientspecific human-induced pluripotent stem cells (hiPSCs) carrying a sarcomere gene variant from a patient with a severe phenotype requiring heart transplantation, to demonstrate the pathogenicity of variants and characterize the molecular events leading to LVNC.

\section{METHODS}

An expanded "Methods" section is available in the Supplemental Appendix. For in vitro experiment, analyses were repeated at least three times for each experiment.

Subjects

Between 2001 and 2014, 120 unrelated Japanese probands with LVNC were referred to our institution, from multiple Japanese

\footnotetext{
${ }^{1}$ Department of Pediatrics, University of Toyama, Toyama, Japan; ${ }^{2}$ Department of Legal Medicine, University of Toyama, Toyama, Japan; ${ }^{3}$ Department of Pediatrics (Cardiology), Shengjing Hospital of China Medical University, Shenyang, China; ${ }^{4}$ Department of Stem Cell Growth and Differentiation, Center for iPS Cell Research and Application (CiRA), Kyoto University, Kyoto, Japan; ${ }^{5}$ Department of Epidemiology and Health Policy, University of Toyama, Toyama, Japan; ${ }^{6}$ Department of Thoracic and Cardiovascular Surgery, University of Toyama, Toyama, Japan and ${ }^{7}$ Department of Pediatrics (Cardiology), University of Utah, Salt Lake City, UT, USA

Correspondence: Fukiko Ichida (fkichida@ctg.u-toyama.ac.jp)

These authors contributed equally: Asami Takasaki, Keiichi Hirono
}

Received: 5 January 2018 Revised: 5 June 2018 Accepted: 27 June 2018

Published online: 6 September 2018 
734

hospitals, for genetic testing. Twelve patients aged over 35 years, 13 patients with known LVNC-associated gene mutations (9 in $T A Z, 2$ in $L D B 3$, and 2 in $S C N 5 A$ ), and 2 patients with known metabolic syndromes were excluded, in order to avoid an overlap of targeted genes and method of genetic analysis between this and our previous study. ${ }^{6}$ The major purpose of this study is to investigate the effects of sarcomere gene variants on LVNC pathology and morbidity.

In addition, patients with congenital heart diseases (CHDs) that induce significant hemodynamic changes, or patients with insufficient clinical information were excluded. No patients had signs or symptoms of neuromuscular disorders. Patients were diagnosed with LVNC by the criteria published by Ichida et al: ${ }^{3}$ (1) LV hypertrophy with deep endomyocardial trabeculations predominantly in the distal portion (apex) of the LV, and distributed over the one wall segment of the LV, (2) a two-layered endocardium with a noncompacted to compacted ratio of $\geq 2.0$, and (3) deep recesses filled with blood from the ventricular cavity visualized on color Doppler imaging. In addition, a dilated cardiomyopathy (DCM) phenotype was diagnosed by echocardiography based on the criteria of Jefferies et al. ${ }^{9}$ DCM was echocardiographically defined as a combination of abnormal LV function (LV ejection fraction (LVEF) $z$-score $<-2$ ) and LV dilation (LV end-diastolic dimension (LVEDD) $z$-score $>2$ ) or the presence of at least moderately depressed LV function, defined as LVEF $z$ score $<-3$ regardless of LV size. Informed consent was obtained from all patients or their parents, according to institutional guidelines. This study protocol conforms to the ethical guidelines of the 1975 Declaration of Helsinki as reflected in a priori approval by the Research Ethics Committee of University of Toyama, Japan.

\section{Genetic analysis}

Genomic DNA was extracted from whole blood using a QuickGene DNA Whole Blood Kit S (Kurabo, Osaka, Japan). Mutation screening was performed on genomic DNA samples for seven genes encoding sarcomere proteins, MYH7, MYBPC3, TNNT2, cardiac troponin I type3 (TNNI3), cardiac troponin C type1 (TNNC1), ACTC1, and TPM1. Primers were designed to amplify across the entire coding sequence of these genes in an exon-by-exon manner. The nucleotide sequences of the amplified fragments were then analyzed by direct sequencing in both directions using a BigDye Terminator v3.1 Cycle Sequencing Kit (Applied Biosystems, Foster city, CA, USA). Sequence analysis was performed using an $A B I$ 3100 automated sequencer (Applied Biosystems, Foster city, CA, USA).

Novel missense variants were considered to be likely pathogenic on the basis of following criteria, based on ACMG guidelines: ${ }^{10}(1)$ it was present in $<0.2 \%$ of ethnicity/race-matched individuals in public databases, JPT (Japanese in Tokyo) and the 1000 Genome Project (1000genomes.org); (2) not reported in the public databases, dbSNP database (http://www.ncbi.nlm.nih.gov/ projects/SNP/), and NHLBI GO Exome Sequencing Project (http:// evs.gs.washington.edu/EVS/); (3) affects evolutionarily conserved residues (nucleotide and amino acid); (4) predicted deleterious/ pathogenic by at least five of seven in silico algorithms (FATHMM (fathmm.biocompute.org), Align GVGD (agvgd.iarc.fr), SIFT (sift. jcvi.org), Polyphen-2 (genetics.bwh.harvard.edu/pph2), Mutation Taster (mutationtaster.org), PROVEAN (provean.jcvi.org), and CADD (cadd.gs.washington.edu) (Supplemental Table S1); and/or (5) has been previously reported to be associated with cardiomyopathy. Truncating mutations were also considered pathogenic.

\section{Immunocytochemistry}

Undifferentiated hiPSC colonies were fixed with 4\% paraformaldehyde (Wako, Japan), stained with primary antibodies for TRA-160 (BD Bioscience), and visualized with secondary antibodies, as previously described. ${ }^{11}$ Dissociated cardiomyocytes differentiated from hiPSCs were fixed with $4 \%$ paraformaldehyde and stained with troponin T (cTnT, 1:2000, Sigma, Japan), MYBPC (1:200, sc166081, Sigma, Japan), and TPM1 (1:200, sc-73225, Thermo Fisher Scientific, Japan) primary antibodies, followed by labeling with secondary antibodies. The cells were mounted with Vectashield (Vector Laboratories Inc., Burlingame, CA, USA) containing 4',6diamidino-2-phenylindole (DAPI) for the nuclei staining and imaged with a Keyence BZ-9000 microscope (Keyence, Japan).

\section{Differentiation of cardiomyocytes}

Cardiomyocyte differentiation was induced as previously reported. ${ }^{12}$ Cells were dissociated with TrypLE Select (Thermo Fisher Scientific, Japan) and seeded onto Matrigel-coated plates and grown in mTeSR1 medium (Veritasm, Japan) plus basic fibroblast growth factor (bFGF) for 2-3 days prior to induction. Cells were covered with Matrigel on the day before induction. For cardiac differentiation, the culture medium was replaced with mouse embryonic fibroblast-conditioned media with RPMI + B27 medium (RPMI-1640, 2 mM L-glutamine, x1 B27 supplement without insulin), supplemented with Activin A (R\&D Systems, Japan) for $24 \mathrm{~h}$. Then, human bone morphogenetic protein 4 (R\&D, Japan) and bFGF were added to the medium. After 4 days, the culture medium was changed to RPMI + B27 without supplementary cytokines and then replaced every 2 days. Beating cells were observed by day 14 .

\section{RNA microarray}

RNA expression profiling was performed using $130 \mathrm{ng}$ of total RNA, and a microarray (Affymetrix GeneChip Human Genome U133 Plus 2.0 Array) according to the manufacturer's instructions. The microarray analyzes 47,000 human messenger RNA (mRNA) transcripts.

\section{$\mathrm{Ca}^{2+}$ imaging}

Clusters of cardiomyocytes (CMs) were dissociated, plated on $0.1 \%$ gelatin-coated coverslips, and cultured for 1 week. To study the $\mathrm{Ca}^{2+}$ handling properties of hiPSC-derived CMs, cells were loaded with $4 \mu \mathrm{M}$ Fluo-8 AM (AAT-Bioquest) for $30 \mathrm{~min}$ in a perfusate medium. The perfusate medium consisted of $115 \mathrm{mM} \mathrm{NaCl}$, $5.4 \mathrm{mM} \mathrm{KCl}, 20 \mathrm{mM}$ HEPES, $13.8 \mathrm{mM}$ D-glucose, and $1.8 \mathrm{mM} \mathrm{CaCl}_{2}$ dissolved in $\mathrm{H}_{2} \mathrm{O}$ and adjusted to 7.4 with $\mathrm{NaOH}$. The fluorescent probe was excited at $490 \mathrm{~nm}$ (Argon) and emissions were collected at 500-550 nm. Images were obtained using a Keyence BZ-9000 microscope (Keyence, Japan) equipped with an argon laser of $488 \mathrm{~nm}$ at a frequency of $59 \mathrm{~Hz}$. Images were acquired in the line-scan (X-T) mode with 512 pixels at a rate of $3 \mathrm{~ms}$ per scan. ${ }^{13,14}$ Data analysis was performed with a BZ-II Analyzer (Keyence, Japan) and VW-9000 Motion Analyzer (Keyence). The distribution of CMs in different categories is presented for each cell line separately.

\section{GO analysis}

Functional annotation analysis of differentially expressed genes was performed using the Database for Annotation, Visualization and Integrated Discovery (DAVID, version 6.8). The ontology consists of three domains: Biological Process, Cellular Component, and Molecular Function. A $p$ value of Gene ontology (GO) terms $\leqq 0.05$ was considered significant.

\section{Statistical analysis}

Univariate analyses were performed using the Student's $t$ test for continuous variables and $x^{2}$ test for categorical variables. Multivariate logistic regression was used to evaluate whether there are differences in the development of severe cardiac outcomes (i.e., implantation of implantable cardioverter-defibrillator (ICD), listing for heart transplantation, and death) between mutation-positive and mutation-negative patients. The odds ratios (ORs) and 95\% 
Sarcomere gene variants act as a genetic trigger underlying the... A Takasaki et al.

confidence intervals (95\% Cls) were calculated after adjusting simultaneously for potential confounders such as age at diagnosis, sex, duration of follow-up, symptom at diagnosis, complications, congenital heart anomaly, and family history. Event-free rates for poor cardiac outcomes were calculated using the Kaplan-Meier method, and the distributions of time-to-event by phenotype group were compared using the log-rank test. $P<0.05$ was considered statistically significant. Statistical analyses were performed using PASW Statistics 18 (SPSS Inc., Chicago, IL, USA) and GraphPad Prism6 (Graph Pad Software, San Diego, CA, USA).

\section{RESULTS}

Patient characteristics

Eighty-two patients, 46 males and 36 females, were included in the present study: 22 were also described in a previous study. ${ }^{6}$ However, there are significant differences between the two studies in terms of age distribution of the study cohort and that most of the patients described in the previous study were recruited later than the patients included in this study (Supplemental Table S2). The clinical characteristics of the patients are described in Table 1. The age at onset/diagnosis ranged from 0 to 35 years old (median: 0.79). The mean follow-up period was 4.82 years $(0-22)$. Twenty-four (29\%) patients reported a family history (with any kind of cardiomyopathy). Associated CHDs without significant hemodynamic effects were identified in 14 patients (17.0\%). Small muscular ventricular septal defects were the most frequent (6 of 14). A DCM phenotype was identified in $72 \%$ of subjects, and was more common in the variant-positive group (86.3\%) than the variant-negative group (66.7\%), although this difference was not statistically significant: only three of the variant-positive group did not have DCM and two of these had a family history of sudden death.

Variants in sarcomere genes

The screening of seven sarcomere genes identified 22 different variants in 22 patients (26.8\%) (Supplemental Table S3). Among them, eight variants were reported in our previous study. ${ }^{6}$ The variants were distributed among five genes, with $M Y H 7$ and MYBPC3 the most commonly affected, accounting for $63 \%$ of the variants; ten variants (45.5\%) were detected in $\mathrm{MYH7}$ and four $(18.1 \%)$ in $M Y B P C 3$. These variants are distributed across the myosin heavy chain and myosin-binding protein $\mathrm{C}$ proteins, similar to reports for HCM and DCM (Supplemental Figure S3). Only eight variants $(36.3 \%)$ were detected in the other genes, TPM1, TNNC1, and ACTC1, with three (13.6\%), $3(13.6 \%)$, and two (9\%) variants, respectively. No variants were detected in TNNT2 and TNNI3. No compound or double heterozygous variants were identified in this cohort.

Among eight families with sarcomere gene variants, genetic screening of additional family members was performed in seven (Supplemental Figure S4). Pedigrees F5 to F8 support autosomal dominant inheritance, with reduced penetrance in some cases (F6), while the mode of inheritance could not be established for the other four families. Notably, the majority of the variants (19, 86.3\%) were novel (not previously identified in patients with cardiomyopathies) and all affected conserved amino acid residues: the three (13.6\%) variants previously described had been identified in patients with $\mathrm{HCM}^{15-22}$ (Supplemental Table S3). The evolutionary conservation of the mutated amino acids is shown in Supplemental Figure S5. All mutations, except one inversion, were missense and each of the missense mutations was predicted to be deleterious/pathogenic by at least five of seven in silico algorithms (Supplemental Table S3).

Genetic and phenotypic analysis

A comparison of clinical characteristics between sarcomere variant-positive and sarcomere variant-negative patients was

\begin{tabular}{|c|c|c|c|}
\hline & $\begin{array}{l}\text { Variant- } \\
\text { negative }\end{array}$ & $\begin{array}{l}\text { Variant- } \\
\text { positive }\end{array}$ & $P$ value \\
\hline Patients, $n$ & 60 & 22 & - \\
\hline Male, $n$ & 35 & 11 & 0.617 \\
\hline $\begin{array}{l}\text { Mean age at onset/diagnosis, } \\
\text { years (range) }\end{array}$ & $5.4(0-31)$ & $4.9(0-35)$ & 0.816 \\
\hline $\begin{array}{l}\text { Median age at onset/ } \\
\text { diagnosis, years }\end{array}$ & 0.79 & 0.67 & - \\
\hline $\begin{array}{l}\text { Mean duration of follow-up, } \\
\text { years (range) }\end{array}$ & $4.4(0-22)$ & $6.1(0.1-15.5)$ & \\
\hline \multicolumn{4}{|l|}{ Age at onset, $n(\%)$} \\
\hline Fetus & $6(10)$ & $2(9.1)$ & - \\
\hline 1 day -2 years & $30(50)$ & $11(50)$ & - \\
\hline $2-19$ years & $18(30)$ & $8(36.4)$ & 0.601 \\
\hline $20-35$ years & $6(10)$ & $1(4.5)$ & 0.668 \\
\hline \multicolumn{4}{|l|}{ Presentation at diagnosis, $n$ (\%) } \\
\hline Asymptomatic & $22(36.7)$ & $8(36.4)$ & 0.979 \\
\hline Heart failure & $27(45)$ & $11(50)$ & 0.803 \\
\hline Syncope & $2(3.3)$ & $0(0)$ & - \\
\hline CPA & $2(3.3)$ & $1(4.5)$ & - \\
\hline DCM phenotype & $40(66.7)$ & $19(86.3)$ & 0.998 \\
\hline Initial LVEF, \% (mean) & $42.2 \pm 2.6$ & $43.0 \pm 4.4$ & 0.867 \\
\hline Initial LVDD $z$-score (mean) & $2.16 \pm 0.31$ & $2.51 \pm 0.46$ & 0.541 \\
\hline Complication of $\mathrm{CHD}, n(\%)$ & $12(20)$ & $2(9.1)$ & 0.332 \\
\hline Family history, $n(\%)$ & $16(26.7)$ & $8(36)$ & 0.268 \\
\hline \multicolumn{4}{|l|}{ Complications, $n$ (\%) } \\
\hline Arrhythmia & $16(26.7)$ & $4(18.2)$ & 0.556 \\
\hline Embolization & $3(5)$ & $0(0)$ & 0.560 \\
\hline Adverse outcome, $n(\%)$ & $9(15)$ & $11(50)$ & 0.0027 \\
\hline ICD, HTX, death & $1,0,8$ & $1,8,2$ & \\
\hline \multicolumn{4}{|c|}{$\begin{array}{l}\text { Family history, cardiomyopathy, or sudden death } \\
\text { CPA cardiopulmonary arrest, DCM dilated cardiomyopathy, LVEF left } \\
\text { ventricular ejection fraction, LVDD left ventricular diastolic dimension, } \\
\text { CHD congenital heart disease, ICD implantable cardioverter-defibrillator, } \\
\text { HTX heart transplantation }\end{array}$} \\
\hline
\end{tabular}

performed (Table 1). Variant-positive patients did not differ from those without variants in terms of sex, age at onset/diagnosis, duration of follow-up, LVEF and LVDD $z$-scores at initial presentation, family history of cardiomyopathy or sudden death, and presence of CHD or other complications. There was a significant difference in the incidence of adverse cardiac outcome ( $p=$ 0.0027) including implantable cardioverter-defibrillator (ICD) implantation, listing for heart transplantation or death, between sarcomere variant-positive and variant-negative patients. Although LVEF and LVDD $z$-scores at initial presentation were not different between the two groups, the event-free survival curve shows significantly worse long-term prognosis in the variant positive group: 11 out of the 22 patients $(50 \%)$ underwent ICD implantation $^{1}$ or heart transplantation, ${ }^{8}$ while two patients died 6 years after diagnosis (Supplemental Figure S7, event-free survival curve).

Table 2 shows the association of types of variants with adverse cardiac outcomes after adjusting for potential confounding factors. Variant-positive patients more frequently had adverse cardiac outcomes (50\%) compared to variant-negative patients. Further, patients with variants in low prevalence genes, TPM1, 
Table 2 Associations of types of variant with adverse cardiac outcomes after adjustment for potential confounding factors

\begin{tabular}{llll}
\hline & Total, $n$ & Adverse outcome, $n$ (\%) & Odds ratio (95\% confidence interval) \\
\hline Variant negative & 60 & $9(15)$ & 1 \\
Variant positive (any variant) & 22 & $11(50)$ & $20.3(3.88-106.1)$ \\
Single variant (MYH7, MYBPC3) & 14 & $5(35.7)$ & $10.2(1.64-63.8)$ \\
Single variant (TNNC1, TPM1, ACTC1) & 8 & $6(75.0)$ & $125.3(7.79-2017.9)$ \\
\hline
\end{tabular}

The model is adjusted for sex, age at diagnosis, duration of follow-up, symptom of heart failure at diagnosis, complications (arrhythmia, embolization), congenital heart anomaly, and family history

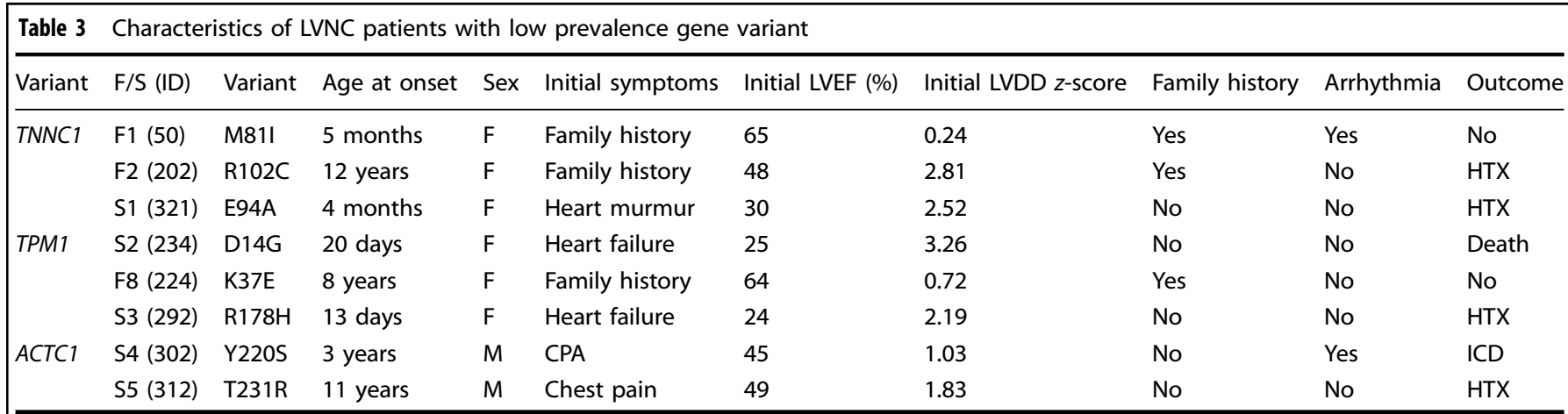

F/S numbers are the same as in Supplementary Figure S4

CPA cardiopulmonary arrest, ICD implantable cardioverter-defibrillator, $H T X$ heart transplantation, $S$ sporadic, $F$ familiar

C

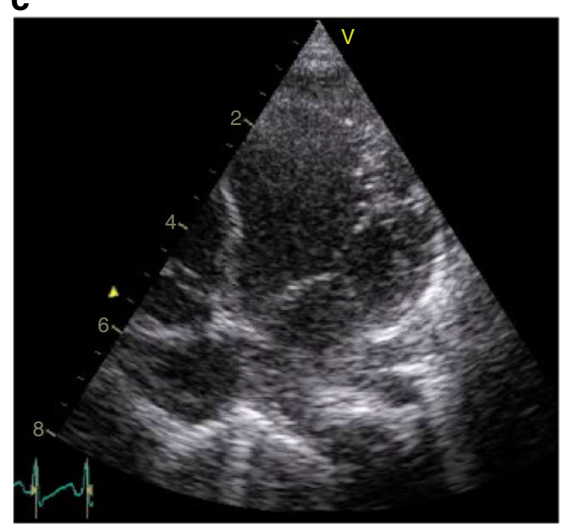

b

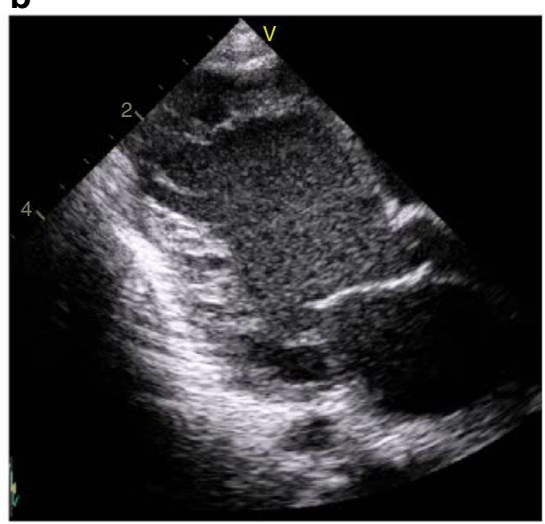

C

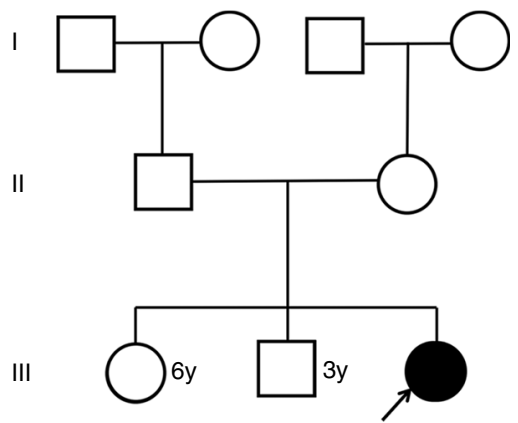

Fig. 1 Echocardiographic images and pedigree of the family. a Apical four-chamber view showing characteristic left ventricular myocardium. b Longitudinal axis view showing characteristic left ventricular myocardium. c Pedigree of the family. The proband is indicated by the arrow. Solid symbols indicate the individual with LVNC; open symbols, unaffected status

TNNC1, or ACTC1, were more likely to have severe adverse outcomes than those with MYH7 or MYBPC3 variants $(75 \%$ and $35.7 \%$, respectively), including four patients requiring heart transplantation, one who died and one requiring ICD insertion (Table 3): detailed clinical information of those patients have been reported elsewhere. ${ }^{15-17}$ Of note, each of the mutations in TPM1, TNNC1, or ACTC1 was novel.

Analysis of hiPSC-derived cardiomyocytes

Patient S3 (ID = 292) had a diagnosis of LVNC because of severe heart failure at the age of 13 days. Her symptoms improved initially upon medical treatment, but the heart failure subsequently worsened and she underwent heart transplantation at
1 year old (Fig. 1). To elucidate the pathological role of variants in sarcomere genes, we applied further analysis using hiPSC-derived cardiomyocytes from this patient (S3), as well as two age-matched, sex-matched, and ethnicity-matched healthy controls (Fig. 2). The pluripotent characteristics of the hiPSCs are illustrated in Supplementary Figure S6.

All the cell lines used in the present study differentiated into cardiomyocytes with similar characteristics. Beating cells appeared at days 10-12 and the beating area spread by day 14 after induction: there was no difference between the lines when the beating areas appeared. After cardiac differentiation, beating clusters were dissociated into single cells and cultured for 1 week. Loss of sarcomere architecture was observed in isolated 
a

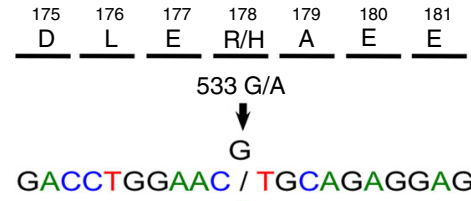

GACCTGGAAC / TGCAGAGGAG A

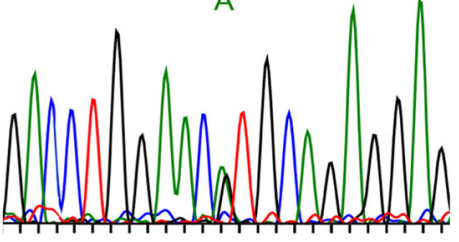

Sequence analysis of TPM1 reveals a change of arginine to histidine at position 178
C

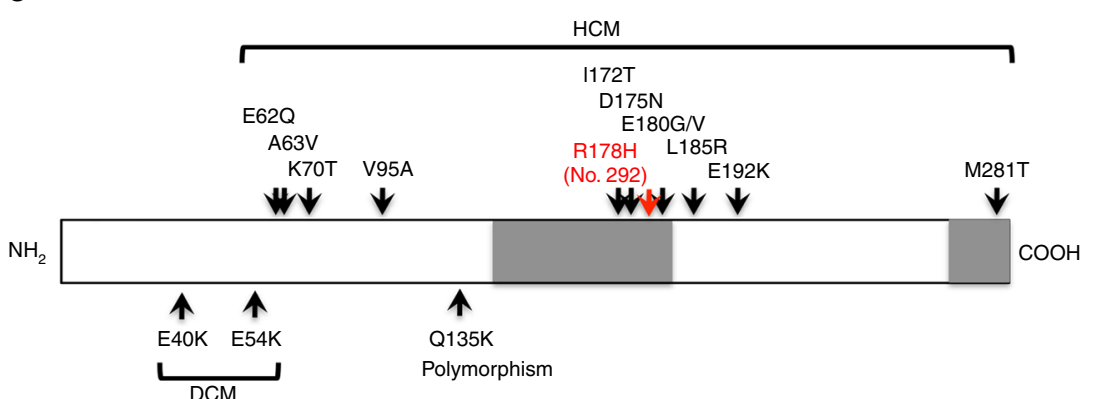

Position of mutations in the primary structure of $\alpha$ tropomyosin protein. The gray boxes represent the putative regions in of $\alpha$-tropomyosin protein that bind troponin-T.

b

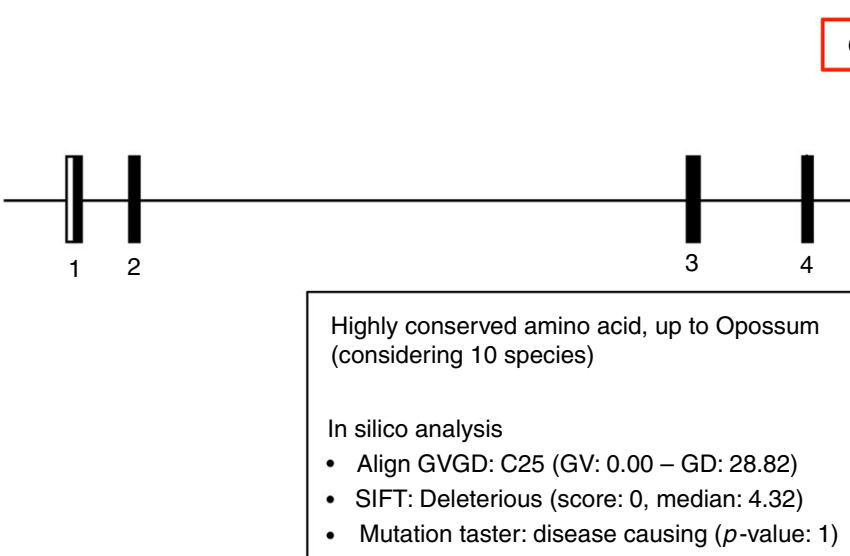

Fig. 2 Sequence analysis of TPM1. a Sequence analysis of TPM1 reveals a change of arginine to histidine at position 178 where it is a highly conserved amino acid, up to opossum (considering 10 species). b In silico analysis showed that the TPM1 gene mutation was pathogenic; Align GVGD: C25 (GV: 0.00, GD: 28.82), SIFT: deleterious (score: 0, median: 4.32), Mutation Taster: disease causing ( $p$ value: 1). c Position of mutations in the primary structure of $\alpha$-tropomyosin protein. The gray boxes represent the putative regions of $\alpha$-tropomyosin protein that bind troponin T. HCM hypertrophic cardiomyopathy, DCM dilated cardiomyopathy

cardiomyocytes derived from the LVNC patient (Fig. 3). Thus, it appears that the TPM1 mutation resulted in mislocalization of tropomyosin 1 and may cause the disruption of the sarcomere structure in cardiomyocytes.

TPM1 mutation affected calcium handling in hiPSC-CMs The $\mathrm{Ca}^{2+}$ handling properties of hiPSC-derived CMs were studied by $\mathrm{Ca}^{2+}$ imaging. TPM1-p.Arg178His-CMs showed significant differences from control CMs (Fig. 4), with lower amplitudes for calcium transients at all time points, but there were no differences in time to peak and frequency of calcium transients (Fig. 4).

\section{$\mathrm{GO}$ analysis}

To identify genes whose expression may have changed due to the presence of the TPM1 variant, we extracted mRNA for microarray analysis from TPM1-Arg178His-derived and control hiPSC-derived CMs. Of 47,000 genes tested, 90 showed more than a 10 -fold decrease in the patient-derived cells compared to the controls (Fig. 5). GO analysis of the 90 genes, using the DAVID web-based integrative knowledge base, ${ }^{18}$ revealed that the major classes of genes identified are those involved in heart development (12 genes, $p<0.05)$, regulation of multicellular organismal process ( 12 genes, $p<0.05)$, and positive regulation of cellular process $(12$ genes, $p<0.05)$ (Fig. 5 and Table 4), almost all of which are highly expressed in the heart and have been shown to regulate cardiac development and remodeling.

Pathway analysis

The top 15 signaling pathways involving down-regulated transcripts are shown in Table 4 and Fig. 6 . The most highly enriched signal pathway is "cardiac muscle contraction." Amongst these pathways, the calcium signaling pathway was found to be involved.

\section{DISCUSSION}

Genotype-phenotype correlations in young patients with LVNC This study revealed a distinct spectrum of sarcomere gene variants in young Japanese patients with LVNC, compared to previous studies in patients with hypertrophic cardiomyopathy: ${ }^{19,20}$ variants in MYH7 appear to be a significant cause of LVNC (45.5\%), while the prevalence of $M Y B P C 3$ variants was unexpectedly low (18.1\%). In addition, most of the variants in our LVNC patients are novel. Patients with variants in genes that are more rarely mutated in cardiomyopathy patients, TPM1, TNNC1, or ACTC1, had poor prognoses in our study (Tables 2 and 3). There are few data describing the clinical course of LVNC in patients with variants in these genes. ${ }^{6,21}$ In a study by Tian et al., ${ }^{21}$ two patients with TPM1 and TNNT2 variants presented with severe heart failure, while the 

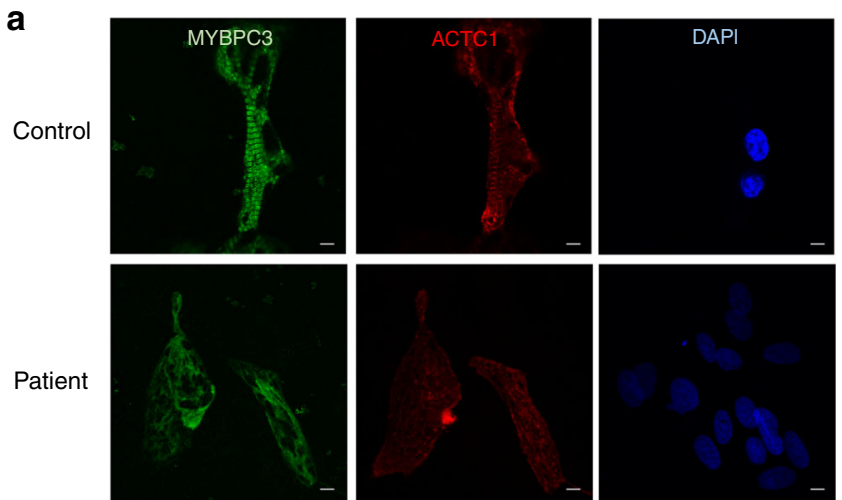

C
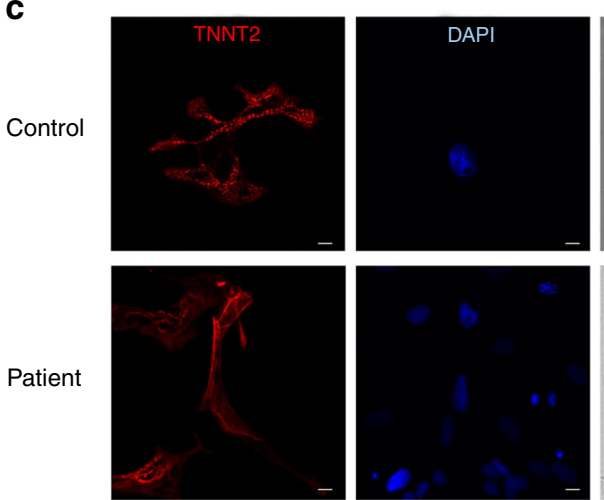

b
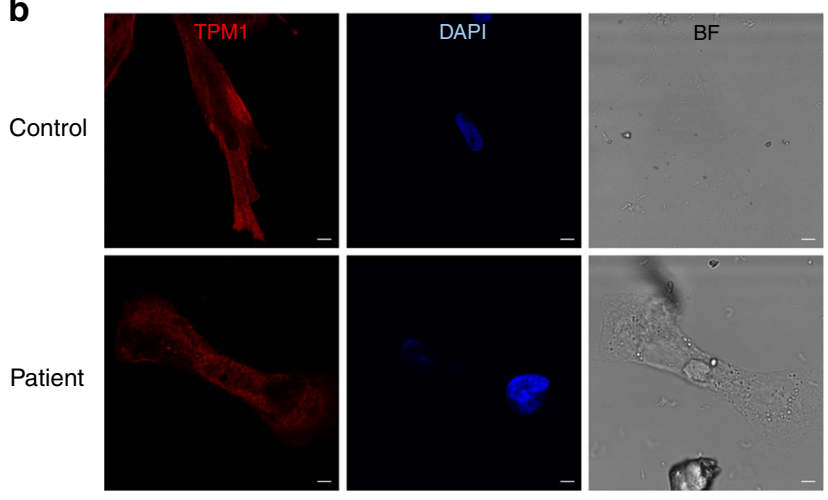

Fig. 3 Tropomyosin 1 and cardiac troponin T colocalize in human iPSC-derived cardiomyocytes. Confocal microscopic images of cardiomyocytes that dissociated into single cells, and were cultured for 1 week after 14 days of differentiation from hiPSCs. a Representative images of TPM1-Arg178His-CMs and control CMs stained with antibodies for myosin-binding protein C (green) and cardiac muscle $\alpha$-actin (red). Nuclei were detected with DAPI staining (blue). Scale bars are $10 \mu \mathrm{m}$. BF bright field. b Representative images of TPM1-Arg178His-CMs and control CMs stained with antibodies for tropomyosin (red). Nuclei were detected with DAPI staining (blue). Scale bars are $10 \mu \mathrm{m}$. c Representative images of TPM1-Arg178His-CMs and control CMs stained with antibodies for cardiac troponin T (red). Nuclei were detected with DAPI staining (blue). Scale bars are $10 \mu \mathrm{m}$

patient with an ACTC1 variant presented with syncope and palpitation, ventricular fibrillation, atrial fibrillation, and left bundle branch block. To date, only one mutation has been reported in TNNC1 in a patient with LVNC, ${ }^{22}$ and are rarely described in HCM or DCM patients: ${ }^{23-29}$ no genotype-phenotype correlations have been reported.

We have previously reported the prenatal diagnosis of LVNC during the second trimester, associated with a novel variant in MYH7: this patient is included in this study. ${ }^{8}$ Sarcomere gene variants may alter normal maturation and function of sarcomere, which is crucial during cardiac development and LV compaction during fetal life. The potential deleterious effect of altered sarcomere proteins may result in "dominant-negative" effects, and even greater susceptibility to the most severe phenotypes, as seen in patients with single mutations in TPM1, TNNC1, and ACTC1.

The recent report of a large cohort from the Pediatric Cardiomyopathy Registry revealed that a specific LVNCassociated cardiomyopathy phenotype, DCM, predicts the risk of death or transplantation. Genetic data were not reported for these patients. ${ }^{9}$ In our cohort, although LVEF and LVDD $z$-scores at initial presentation were not different between the two groups, the event-free survival curve shows a significantly worse long-term prognosis in the variant-positive group; most of variant-positive patients gradually developed heart failure due to decreased LVEF over time (Supplement S7). Therefore, the deterioration of sarcomere function may have a pivotal role in inducing depressed LV function after birth, leading to the poor prognosis. Our findings suggest that genetic testing should be considered for individuals with LVNC with reduced LV function and a DCM phenotype, since genetic information may be useful in providing earlier planning for advanced treatment and predict the need for heart transplantation.

Sarcomere mutation-specific phenotypes in hiPSC-derived cardiomyocytes

We derived hiPSCs from a patient carrying a Japanese LVNC founder mutation in TPM1 (p.Arg178His). hiPSC-derived cardiomyocytes carrying this mutation displayed changes consistent with the pathological phenotype, specifically mislocalization of tropomyosin 1, resulting in the disruption of the sarcomere and impaired calcium handling. In addition to morphological and functional differences, gene expression profiles were different between these and control CMs, including many genes involved in heart development and regulation of multicellular organismal process, and positive regulation of cellular process, especially in calcium signaling pathways.

Tropomyosin coordinates $\mathrm{Ca}^{2}$-mediated changes in the structure of the troponin complex with myosin-mediated displacement of the actin filament. It has been observed that cardiomyopathycausing mutations in tropomyosin affect $\mathrm{Ca}^{2}$ sensitivity, ${ }^{30}$ likely by inducing conformational changes in the troponin complex. ${ }^{31}$ In addition, tropomyosin is a critical element in the cooperative binding of myosin to actin. We, therefore, postulate that studying a relatively unexplored tropomyosin mutant could yield novel insights into mechanisms that regulate cardiac contraction in LVNC. 
a

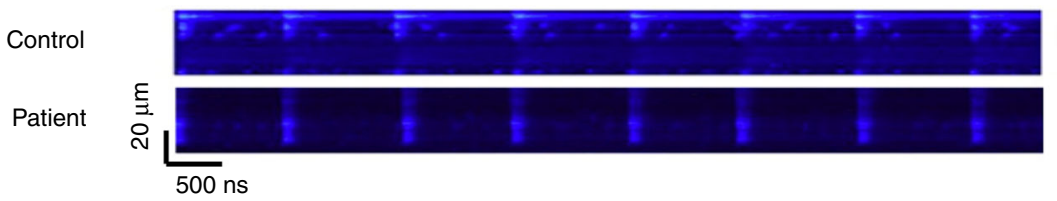

b

Control
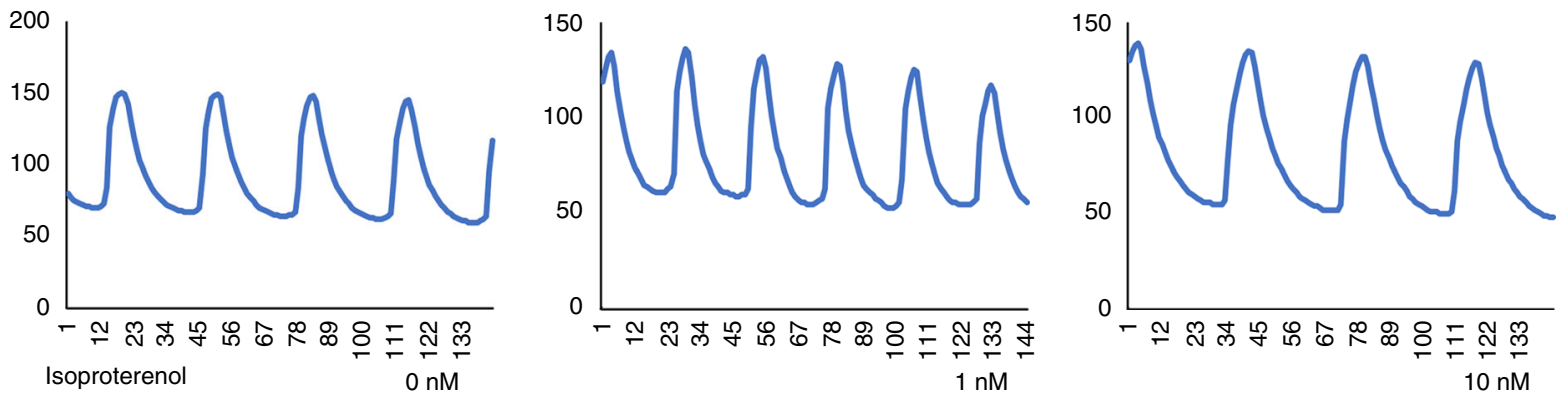

Patient
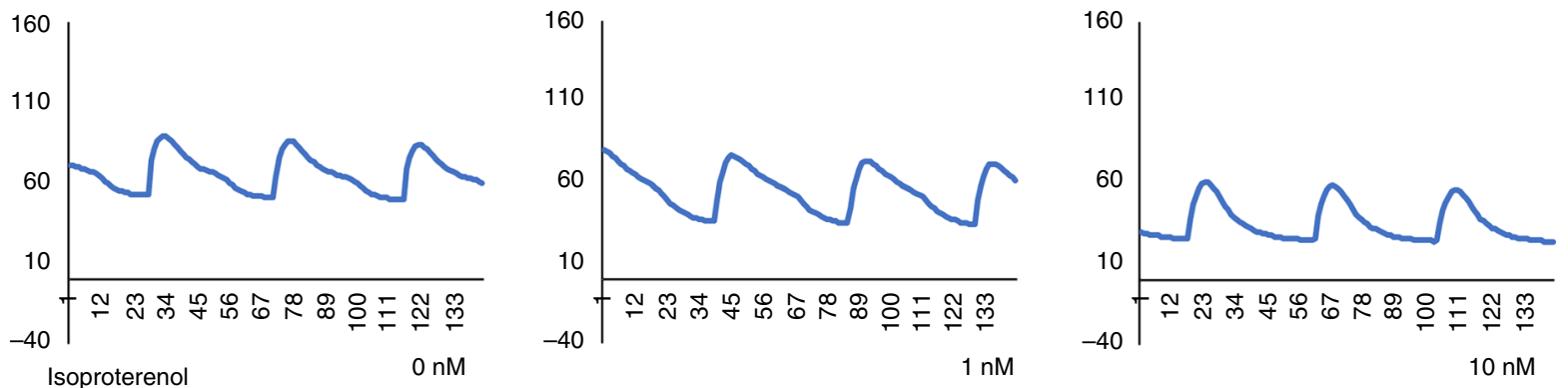

Fig. 4 TPM1-Arg178His decreased the calcium handling of hiPSC-CMs in a persistent manner. a Two representative line-scan (X-T mode) images of (local Ca2 ${ }^{+}$release) LCR and $\mathrm{Ca}^{2+}$ waveforms of hiPSC-CMs. b Raw traces of spontaneous calcium transients (CaTs) at basal level and upon treatment with 1 and $10 \mathrm{nM}$ isopropanol (as control) in hiPSC-CMs. c Summarized data on amplitude, time-to-peak, and frequency of calcium transients (CaTs) upon treatment with 1 and $10 \mathrm{nM}$ isopropanol (as control) in hiPSC-CMs. Values are mean \pm SEM of four to six independent experiments. ${ }^{*} P<0.05,{ }^{* *} p<0.01$, and ${ }^{* * *} p<0.001$. NA not applicable, Isp isopropanol

Point mutations in tropomyosin can also affect the energy landscape of tropomyosin interactions with actin, providing another potential route for mutations to affect thin filament calcium regulation. ${ }^{31}$ Altered actin binding by mutant tropomyosin is also supported by numerous experimental and computational studies. ${ }^{32,33}$ Changes in HCM/DCM-related tropomyosin flexibility are widely thought to affect thin filament cooperativity. ${ }^{34,35}$ Our data suggest TPM1 p.Arg178His results in a muscle intrinsically capable of producing less force at any activating $\mathrm{Ca}^{2}$ concentration. Left ventricular dilation may represent a compensatory mechanism to maintain stroke volume in the setting of reduced contractility as the result of decreased force production and/or $\mathrm{Ca}^{2}$ affinity associated with the TPM1 mutation. In addition, our results suggest that TPM1 mutations may cause differences in protein stability, actin binding, and sarcomere conformation. All of these differences could converge to change the $\mathrm{Ca}^{2}$ dependence of myosin activity. The common phenotype of mutations in sarcomeric proteins with related functions suggests that for this subset of LVNC-causing mutations there could be a common causative molecular mechanism. This finding demonstrated a unique and poorly understood mechanism linking sarcomeric dysfunction to LVNC.

\section{Limitations}

The present study was conducted over about 15 years and during that time disease-modifying treatments have been developed that may have altered outcomes. The small number of patients with variants in low-frequency genes limits the significance of the relationship between these variants and outcome. Further analysis of larger numbers of patients will be needed to clarify the impact of sarcomere gene variants on clinical phenotype and prognosis. In addition, functional analyses are needed to determine how each of the detected variants contribute to the pathogenesis of LVNC, with one possibility being the use of patient-specific iPSCs and their differentiated cardiomyocytes (iPSC-CMs), especially for low-prevalence genes such as TNNC1 and ACTC1. The majority of the sarcomere variants reported in this study are novel. Therefore, these variants may have different effects on sarcomere protein production and likely cause differences in cardiomyopathic phenotype. Further studies specifically addressing the LVNC phenotype of extensive trabeculation and/or non-compaction would be warranted. Further, we did not use an isogenic, mutation-corrected iPSC line as a control for comparison or increase the number of disease and control cell lines for transcriptional comparison.

Another limitation of our study is incomplete clinical family screening and genetic testing, as recommended by the HFSA Practice Guidelines. ${ }^{36}$ Clinical and echocardiographic screening would more clearly identify genetic inheritance patterns and penetrance of this disease, and should be considered to detect asymptomatic family members with potential risk for arrhythmias, myocardial dysfunction, and thromboembolic events. 


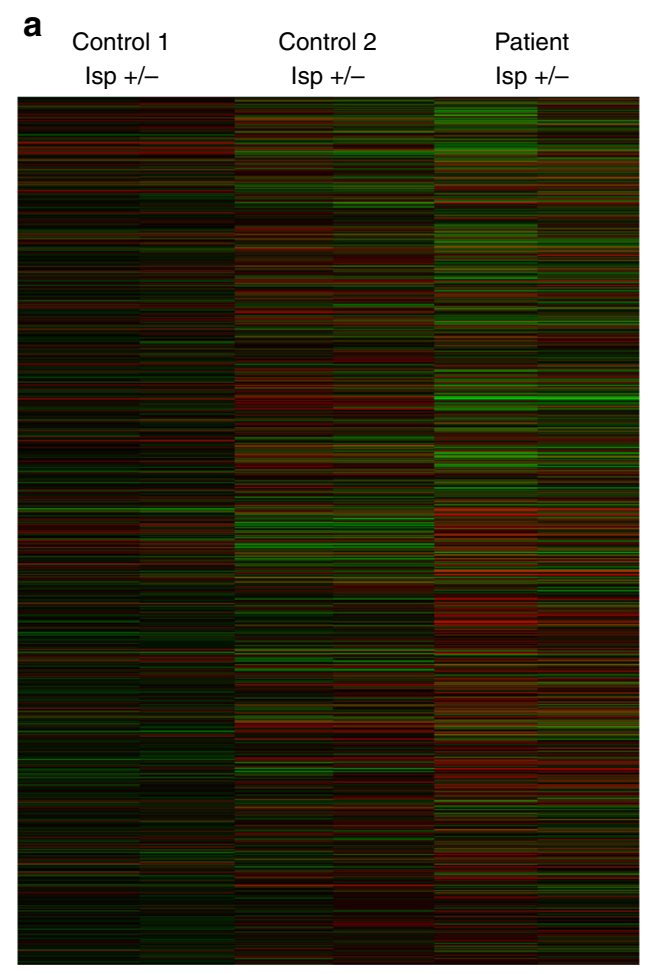

\begin{tabular}{lrc}
\hline Gene symbol & $\begin{array}{c}\text { Fold change }\left(\log _{2}\right) \\
\text { patient vs control } 1\end{array}$ & $\begin{array}{c}\text { Fold change }\left(\log _{2}\right) \\
\text { patient vs control } 2\end{array}$ \\
\hline MYH6/IIMYH7 & -9.61 & -10.40 \\
TECRL & -9.50 & -9.43 \\
MYH6 & -9.41 & -9.70 \\
WT1 & -8.81 & -5.60 \\
TNNT2 & -8.67 & -9.49 \\
MYOZ2 & -8.35 & -10.05 \\
GATA6 & -8.21 & -8.03 \\
SLC8A1 & -7.70 & -8.97 \\
EDNRA & -7.44 & -5.54 \\
FILIP1 & -7.32 & -8.12 \\
SYNPO2L & -7.31 & -8.15 \\
UNC45B & -7.29 & -8.61 \\
MYLK3 & -7.10 & -8.24 \\
ACTN2 & -6.93 & -8.09 \\
FILIP1 & -6.88 & -7.80 \\
MYOZ2 & -6.81 & -8.66 \\
ZNF385B & -6.77 & -6.55 \\
GATA4 & -6.70 & -7.00 \\
BMP5 & -6.63 & -7.18 \\
TRIM55 & -6.60 & -7.33 \\
NBLAO0301 & -6.56 & -8.45 \\
VWDE & -6.47 & -6.69 \\
TTN & -6.42 & -7.10 \\
\hline & &
\end{tabular}

Fig. 5 mRNA array data from TPM1-Arg178His-CMs and control CMs. a Heat maps and hierarchical clustering of expression ratios (log 2 scale) of mRNAs in TPM1-Arg178His-CMs and control CM. "Red" denotes high relative expression and "blue" denotes low relative expression. b mRNAs extracted from TPM1-Arg178His-CMs and control CMs showed that some of the mRNAs of TPM1-Arg178His-CMs are downregulated when compared to that of control

\begin{tabular}{|c|c|c|c|c|}
\hline BP & Heart development & 12 & $3.40 \mathrm{E}-10$ & $\begin{array}{l}\text { GATA4, GATA6, TBX5 EDNRA, ITGB1, MIB1, MB, MYBPC3, MYH6, TTN, } \\
\text { TNNI3, TNNT2 }\end{array}$ \\
\hline BP & $\begin{array}{l}\text { Regulation of multicellular organismal } \\
\text { process }\end{array}$ & 12 & $6.00 \mathrm{E}-04$ & $\begin{array}{l}\text { GATA4, GATA6, TBX5, EDNRB, Hey, MIB1, MYBPC3, MYH6, RYR2, SLC8A1, } \\
\text { TNNI3, TNNT2 }\end{array}$ \\
\hline BP & Positive regulation of cellular process & 12 & $7.90 \mathrm{E}-02$ & $\begin{array}{l}\text { GATA4, GATA5, GATA6, BST2, TBX5, WT1, EDNRA, EDNRB, Hey2, ITGB1, MIB1, } \\
\text { RYR2 }\end{array}$ \\
\hline BP & Regulation of biological quality & 10 & $9.50 \mathrm{E}-02$ & CORIN, EDNRA, EDNRB, JPH2, MB, MYH6, QK1, RYR2, SLC8A1, TNNI3 \\
\hline BP & Muscle organ development & 9 & $1.10 \mathrm{E}-06$ & GATA6, TBX5, ITGB1, MYBPC3, MYH6, TTN, TNNI3, TNNT2, UNC45B \\
\hline BP & Regulation of molecular function & 9 & $2.70 \mathrm{E}-02$ & EDNRA, EDNRB, JPH2, MYBPC3, MYH6, RUNX1T1, TTN, TNNI3, TNNT2 \\
\hline BP & Striated muscle contraction & 8 & $3.30 \mathrm{E}-10$ & KLHL41, MB, MYOM1, MYBPC3, MYH6, RYR2, TTN, TNNI3 \\
\hline BP & Embryonic development & 8 & $5.10 \mathrm{E}-03$ & GATA4, GATA6, TBX5, EDNRA, HOXB3, ITGB1, MIB1, MYH6 \\
\hline BP & Homeostatic process & 8 & $2.10 \mathrm{E}-02$ & EDNRA, EDNRB, JPH2, MB, QK1, RYR2, SLC8A1, TNNI3 \\
\hline BP & Heart morphogenesis & 7 & $2.90 \mathrm{E}-07$ & GATA4, MIB1, MYBPC3, MYH6, TTN, TNNI3, TNNT2 \\
\hline BP & Muscle cell differentiation & 7 & $5.80 \mathrm{E}-06$ & GATA6, TBX5, ITGB1, MYH6, QK1, TTN, TNNT2 \\
\hline
\end{tabular}

\section{CONCLUSIONS}

Poor prognoses were observed in patients with sarcomere gene variants, especially in low-frequency-variant genes, TPM1, TNNC1, or ACTC1. Our study using iPSC-CMs indicate that sarcomere genes act as genetic triggers in LVNC, regulating the expression of numerous genes involved in heart development resulting in imbalanced cardiomyocyte proliferation and/or differentiation processes. In addition, sarcomere genes may cause differences in protein stability, actin binding, and sarcomere conformation, and underlie variations in the severity of disease. 


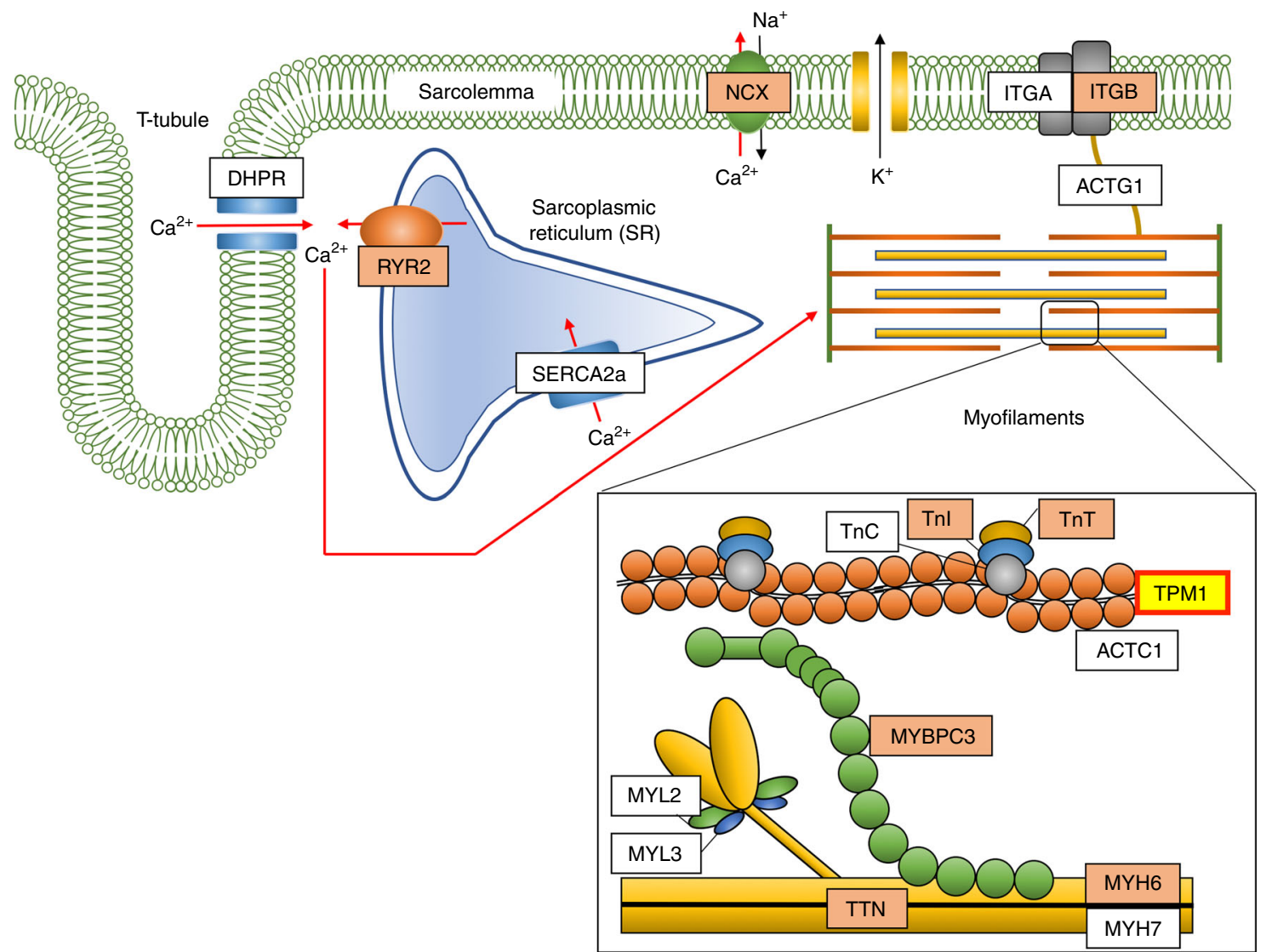

Fig. 6 Pathway analysis is a functional analysis mapping genes to KEGG pathways. The schematic diagram of the gene category "cardiac muscle contraction." Nodes marked in yellow are associated with down-regulated genes. Orange marked nodes are associated with upregulated or only whole dataset genes, while white nodes have no significance

\section{ACKNOWLEDGEMENTS}

We gratefully acknowledge W, Chung, L.J. Addnizio, and all Noncompaction study collaborators (Supplement S8). We are also grateful to the patients and families for their participation and to the referring clinicians for supporting the study. We thank Professor Yuichi Adachi for the steadfast counsel and guidance. This work was supported by grants from the Ministry of Education, Culture, Sports, Science, and Technology in Japan (Research Project Number: 15K09685, 24591571, and 17591072) and by Japan Heart Foundation Research Grant on Dilated Cardiomyopathy to F.I.

\section{ADDITIONAL INFORMATION}

The online version of this article (https://doi.org/10.1038/s41390-018-0162-1) contains supplementary material, which is available to authorized users.

Competing interests: The authors declare no competing interests.

Publisher's note: Springer Nature remains neutral with regard to jurisdictional claims in published maps and institutional affiliations.

\section{REFERENCES}

1. Chin, T. K., Perloff, J. K., Williams, R. G., Jue, K. \& Mohrmann, R. Isolated noncompaction of left ventricular myocardium. A study of eight cases. Circulation $\mathbf{8 2}$, 507-513 (1990).

2. Maron, B. J. et al. Contemporary definitions and classification of the cardiomyopathies: An American Heart Association Scientific Statement from the Council on Clinical Cardiology, Heart Failure and Transplantation Committee; quality of care and outcomes research and functional genomics and translational biology interdisciplinary working groups; and council on epidemiology and prevention. Circulation 113, 1807-1816 (2006).

3. Ichida, F. et al. Novel gene mutations in patients with left ventricular noncompaction or barth syndrome. Circulation 103, 1256-1263 (2001).
4. Klaassen, S. et al. Mutations in sarcomere protein genes in left ventricular noncompaction. Circulation 117, 2893-2901 (2008).

5. Hoedemaekers, Y. M. et al. The importance of genetic counseling, DNA diagnostics, and cardiologic family screening in left ventricular noncompaction cardiomyopathy. Circ. Cardiovasc. Genet. 3, 232-239 (2010).

6. Wang, C. et al. A wide and specific spectrum of genetic variants and genotype-phenotype correlations revealed by next-generation sequencing in patients with left ventricular noncompaction. J. Am. Heart Assoc. 6, e006210 (2017).

7. Nomura, Y. et al. A novel MYH7 gene mutation in a fetus with left ventricular noncompaction. Can. J. Cardiol. 31, 103.e1-3 (2015).

8. Sedmera, D., Pexieder, T., Vuillemin, M., Thompson, R. P. \& Anderson, R. H. Developmental pattern of the myocardium. Anat. Rec. 28, 319-337 (2000).

9. Jefferies, J. L. et al. Cardiomyopathy phenotypes and outcomes for children with left ventricular myocardial noncompaction: Results from the pediatric cardiomyopathy registry. J. Card. Fail. 21, 877-884 (2015).

10. Richards, S. et al. Standards and guidelines for the interpretation of sequence variants: A joint consensus recommendation of the american college of medical genetics and genomics and the association for molecular pathology. Genet. Med. 17, 405-424 (2015).

11. Ojala, M. et al. Culture conditions affect cardiac differentiation potential of human pluripotent stem cells. PLoS ONE 7, e48659 (2012).

12. Uosaki, H. et al. Efficient and scalable purification of cardiomyocytes from human embryonic and induced pluripotent stem cells by VCAM1 surface expression. PLOS ONE 6, e23657 (2011).

13. RJ, Parks \& Howlett, S. E. H-89 decreases the gain of excitation-contraction coupling and attenuates calcium sparks in the absence of beta-adrenergic stimulation. Eur. J. Pharmacol. 691, 163-172 (2012).

14. Mercado, J. et al. Local control of TRPV4 channels by AKAP 150 -targeted PKC in arterial smooth muscle. J. Gen. Physiol. 143, 559-575 (2014).

15. Nihei, K. et al. Wolff-Parkinson-White (WPW) syndrome in isolated noncompaction of the ventricular myocardium (INVM). Circ. J. 68, 82-84 (2004). 
Sarcomere gene variants act as a genetic trigger underlying the...

A Takasaki et al.

742

16. Chang, B. et al. Identification of a novel TPM1 mutation in a family with left ventricular noncompaction and sudden death. Mol. Genet. Metab. 102, 200-206 (2011).

17. Yoshida, Y. et al. A novel ACTC1 gene mutation in a young boy with left ventricular noncompaction and arrhythmias. Heart Rhythm Rep. 2, 92-97 (2016).

18. Huang da, W., Sherman, B. T. \& Lempicki, R. A. Systematic and integrative analysis of large gene lists using DAVID bioinformatics resources. Nat. Protoc. 4, 44-57 (2009).

19. Maron, B. J., Maron, M. S. \& Semsarian, C. Genetics of hypertrophic cardiomyopathy after 20 years: clinical perspectives. J. Am. Coll. Cardiol. 60, 705-715 (2012).

20. Otsuka, H. et al. Prevalence and distribution of sarcomeric gene mutations in Japanese patients with familial hypertrophic cardiomyopathy. Circ. J. 76, 453-461 (2012).

21. Tian, T. et al. A low prevalence of sarcomeric gene variants in a chinese cohort with left ventricular non-compaction. Heart Vessels 30, 258-264 (2015).

22. Miszalski-Jamka, K. et al. Novel genetic triggers and genotype-phenotype correlations in patients with left ventricular noncompaction. Circ. Cardiovasc Genet. 10, e001763 (2017).

23. Landstrom, A. P. et al. Molecular and functional characterization of novel hypertrophic cardiomyopathy susceptibility mutations in TNNC1-encoded troponin c. J. Mol. Cell. Cardiol. 45, 281-288 (2008).

24. Parvatiyar, M. S. et al. A mutation in TNNC1-encoded cardiac troponin c, TNNC1a31s, predisposes to hypertrophic cardiomyopathy and ventricular fibrillation. J. Biol. Chem. 287, 31845-33155 (2012).

25. Chung, W. K., Kitner, C. \& Maron, B. J. Novel frameshift mutation in troponin C (TNNC1) associated with hypertrophic cardiomyopathy and sudden death. Cardiol. Young 21, 345-348 (2011).
26. Mogensen, J. et al. Severe disease expression of cardiac troponin C and T mutations in patients with idiopathic dilated cardiomyopathy. J. Am. Coll. Cardiol. 44, 2033-2040 (2004).

27. Kamisago, M. et al. Mutations in sarcomere protein genes as a cause of dilated cardiomyopathy. N. Engl. J. Med. 343, 1688-1696 (2000).

28. Moller, D. V. et al. The role of sarcomere gene mutations in patients with idiopathic dilated cardiomyopathy. Eur. J. Hum. Genet. 17, 1241-1249 (2009).

29. Herman, D. S. et al. Truncations of titin causing dilated cardiomyopathy. N. Engl. J. Med. 366, 619-628 (2012).

30. Redwood, C. \& Robinson, P. Alpha-tropomyosin mutations in inherited cardiomyopathies. J. Muscle Res. Cell Motil. 34, 285-294 (2013).

31. Orzechowski, M., Fischer, S., Moore, J. R., Lehman, W. \& Farman, G. Energy landscapes reveal the myopathic effects of tropomyosin mutations. Arch. Biochem. Biophys. 564, 89-99 (2014).

32. Janco, M. et al. Alpha-tropomyosin with a D175N or E180G mutation in only one chain differs from tropomyosin with mutations in both chains. Biochemistry 51, 9880-9890 (2012).

33. Zheng, W., Hitchcock-DeGregori, S. E. \& Barua, B. Investigating the effects of tropomyosin mutations on its flexibility and interactions with filamentous actin using molecular dynamics simulation. J. Muscle Res. Cell Motil. 37, 131-147 (2016).

34. Loong, C. K., Zhou, H. X. \& Chase, P. B. Familial hypertrophic cardiomyopathy related $\mathrm{E} 180 \mathrm{G}$ mutation increases flexibility of human cardiac alpha-tropomyosin. FEBS Lett. 586, 3503-3507 (2012).

35. Loong, C. K., Badr, M. A. \& Chase, P. B. Tropomyosin flexural rigidity and single ca regulatory unit dynamics: implications for cooperative regulation of cardiac muscle contraction and cardiomyocyte hypertrophy. Front. Physiol. 3, 80 (2012).

36. Hershberger, R. E. et al. Genetic evaluation of cardiomyopathy-a Heart Failure Society of America Practice Guideline. J. Card. Fail. 15, 83-97 (2009). 